\title{
O uso de microRNAs como biomarcadores do câncer cervical
}

\author{
The use of microRNAs as cervical cancer biomarkers \\ El uso de microRNAs como biomarcadores de cáncer de cérvix
}

Recebido: 14/01/2022 | Revisado: 19/01/2022 | Aceito: 22/01/2022 | Publicado: 24/01/2022

\author{
Priscila Magna do Nascimento Silva \\ ORCID: https://orcid.org/0000-0003-4580-6975 \\ Centro Universitário UNINASSAU, Brasil \\ E-mail: pricilamagna@gmail.com
}

\begin{abstract}
Resumo
Esse trabalho pretendeu descrever o papel dos miRNAs na regulação gênica do câncer cervical. Para esta revisão integrativa de literatura, utilizou-se artigos encontrados nas bases de dados PubMed, SciELO, Lilacs e Sciencedirect, selecionando trabalhos publicados entre 2011 e 2021, na língua portuguesa e inglesa, considerando-se os seguintes descritores: "microRNAs", "regulação da expressão gênica", "câncer de colo do útero" e "biomarcadores". Encontrou-se um total de 156 trabalhos, sendo, por fim, selecionados 17 trabalhos para estudo. Estudos apontaram uma relação direta entre miRNAs e os estágios de desenvolvimento do câncer cervical. Diversos miRNAs, como miR21 e miR-135a, têm sua expressão aumentada em células de câncer cervical quando comparada a seus níveis em indivíduos normais. Outros miRNAs, como miRNA-20a, miR-1246, miR-2392, miR-3147, miR-3162-5p e miR-4484, foram identificados como promissores biomarcadores para deteç̧ão de metástase em pacientes com neoplasia cervical. Já variação nos níveis de expressão de miRNAs pode interferir na sensibilidade à quimioterapia, como o miR-155 que aumenta a quimiossensibilidade à cisplatina, agente antineoplásico. Dessa forma, a análise das assinaturas de miRNAs em pacientes como câncer cervical surge como promissora ferramenta para o seu diagnóstico, prognóstico e tratamento, sendo necessária a associação desses aos métodos usuais para um melhor manejo clínico.
\end{abstract}

Palavras-chave: MicroRNA; Regulação da expressão gênica; Câncer de colo do útero; Biomarcadores.

\begin{abstract}
This work aimed to describe the role of miRNAs in the gene regulation of cervical cancer. For this integrative literature review, articles found in the PubMed, SciELO, Lilacs and Sciencedirect databases were used, selecting works published between 2011 and 2021, in Portuguese and English, considering the following descriptors: "microRNAs", "regulation of gene expression", "cervical cancer" and "biomarkers". A total of 156 works were found, and finally, 17 works were selected for study. Studies have pointed to a direct relationship between miRNAs and the stages of development of cervical cancer. Several miRNAs, such as miR-21 and miR-135a, have their expression increased in cervical cancer cells when compared to their levels in normal individuals. Other miRNAs, such as miRNA-20a, miR-1246, miR-2392, miR-3147, miR-3162-5p and miR-4484, have been identified as promising biomarkers for the detection of metastasis in patients with cervical cancer. Variation in the expression levels of miRNAs, on the other hand, can interfere with sensitivity to chemotherapy, such as miR-155, which increases chemosensitivity to cisplatin, an antineoplastic agent. Thus, the analysis of miRNA signatures in patients such as cervical cancer appears as a promising tool for its diagnosis, prognosis and treatment, requiring their association with the usual methods for a better clinical management.
\end{abstract}

Keywords: MicroRNAs; Regulation of gene expression; Cervical câncer; Biomarkers.

\section{Resumen}

Este trabajo tuvo como objetivo describir el papel de los miRNAs en la regulación génica del cáncer de cuello uterino. Para esta revisión integrativa de la literatura, se utilizaron artículos encontrados en las bases de datos PubMed, SciELO, Lilacs y Sciencedirect, seleccionando trabajos publicados entre 2011 y 2021, en portugués e inglés, considerando los siguientes descriptores: "microRNAs", "regulation of gene expression", "câncer de cuello uterino" y "biomarcadores". Se encontraron un total de 156 obras y, finalmente, se seleccionaron 17 obras para su estudio. Los estudios han demostrado una relación directa entre los miARN y las etapas de desarrollo del cáncer de cuello uterino. Varios miARN, como miR-21 y miR-135a, tienen una expresión aumentada en células de cáncer de cuello uterino en comparación con sus niveles en individuos normales. Otros miARN, como miARN-20a, miR-1246, miR-2392, miR3147, miR-3162-5p y miR-4484, se han identificado como biomarcadores prometedores para la detección de metástasis en pacientes con cáncer de cuello uterino. La variación en los niveles de expresión de miARN puede interferir con la sensibilidad a la quimioterapia, como miR-155, que aumenta la quimiosensibilidad al cisplatino, un agente antineoplásico. Así, el análisis de firmas de miRNA en pacientes con cáncer de cérvix surge como una 
herramienta prometedora para su diagnóstico, pronóstico y tratamiento, requiriendo la asociación de estos con los métodos habituales para un mejor manejo clínico.

Palabras clave: MicroRNAs; Regulación de la expresión génica; Cáncer de cuello uterino; Biomarcadores.

\section{Introdução}

O câncer cervical é uma neoplasia maligna caracterizada pela multiplicação desordenada de células do epitélio de revestimento do órgão, capaz de invadir o tecido subjacente (Vaz et al., 2020). É considerado um problema de saúde pública mundial, tendo a expressão de seus indicadores de incidência, prevalência e mortalidade, aumentada nos países subdesenvolvidos e com baixas condições socioeconômicas (Silva et al., 2020).

Globalmente, o câncer cervical é o quarto tipo de câncer mais comum entre as mulheres, sendo especialmente incidente em países de baixa e média renda, como África do Sul, Índia, China e Brasil (Hull et al., 2020). Pacientes diagnosticadas nas fases iniciais da patologia apresentam resultados clínicos mais favoráveis após tratamento sistêmico, contudo, uma grande parte dos casos são diagnosticados em fases avançadas dada à sua natureza assintomática e inespecífica nas fases iniciais (Marth et al., 2017).

O exame de Papanicolau e a colposcopia são os métodos mais utilizados para detecção do câncer cervical. Mesmo diante da eficácia do uso do exame de Papanicolau no rastreamento e detecção precoce do câncer, sua sensibilidade pode ser variada, gerando resultados falso-negativos. A colposcopia, por sua vez, é um procedimento invasivo e pode gerar custos e riscos adicionais (Qiu et al., 2020).

Ademais, essas metodologias não oferecem informações que permitam a previsão da recorrência e avaliação prognóstica do câncer cervical. Assim, a identificação de novos biomarcadores para diagnóstico e prognóstico é um importante fator para manejo dos pacientes durante o seu diagnóstico e tratamento (Hasanzadeh et al., 2019).

O câncer cervical é causado, principalmente, por tipos de papilomavírus humano (HPV) de alto risco e alterações epigenéticas nas células hospedeiras do vírus possibilitam a invasão tecidual e têm grande potencial como marcadores moleculares na triagem cervical. Dentre essas alterações epigenéticas, se destaca a desregulação da expressão de microRNA (miRNA), frequentemente observada no câncer cervical (Pardini et al., 2018).

Os miRNAs são pequenas moléculas de RNAs não codificadores de fita simples com, aproximadamente, 19-22 nucleotídeos, são altamente conservados e regulam a expressão gênica no nível pós-transcricional. Se ligam à região 3 'não traduzida (3' UTR) do RNA mensageiro (mRNA) alvo para induzir degradação ou repressão traducional (Iorio \& Croce, 2012).

Os miRNAs desempenham papel fundamental em muitas funções biológicas, como proliferação, diferenciação, desenvolvimento e apoptose celular. Além disso, os miRNAs podem atuar como promotores ou supressores de tumores, colaborando para a transformação maligna celular, incluindo o câncer cervical (Iorio \& Croce, 2012; Qiu et al., 2020).

À vista disso, esse trabalho pretende descrever o papel dos miRNAs na regulação gênica do câncer cervical, uma vez que seus perfis podem emergir como promissores biomarcadores tumorais.

\section{Metodologia}

Foi realizada uma revisão integrativa da literatura, que possibilita a síntese de conhecimento e a aplicabilidade dos resultados mais relevantes do estudo, tendo um cunho qualitativo e objetivo exploratório e descritivo, ao realizar um levantamento bibliográfico e correlacionar variáveis encontradas (Souza et al., 2010).

A busca foi feita nas seguintes bases de dados eletrônicos: PubMed, SciELO, Lilacs e Sciencedirect, utilizando trabalhos publicados entre 2011 e 2022. 
Para a seleção dos descritores/termos utilizados, foi realizada consulta ao DECs (descritores de assunto em ciências da saúde da BIREME), considerando-se os seguintes termos e seus correspondentes, na língua portuguesa e inglesa: “microRNAs", "regulação da expressão gênica", "câncer de colo do útero" e "biomarcadores".

Encontrou-se um total de 156 trabalhos, dentre estes 71 encontravam-se repetidos. Na primeira etapa, após leitura do título e do resumo, foram selecionados para leitura do texto na íntegra 38 trabalhos e excluídos 47 trabalhos, a partir dos critérios de inclusão e exclusão. Totalizando-se 17 trabalhos selecionados para estudo (Figura 1).

Figura 1. Fluxograma da seleção de trabalhos para revisão integrativa.

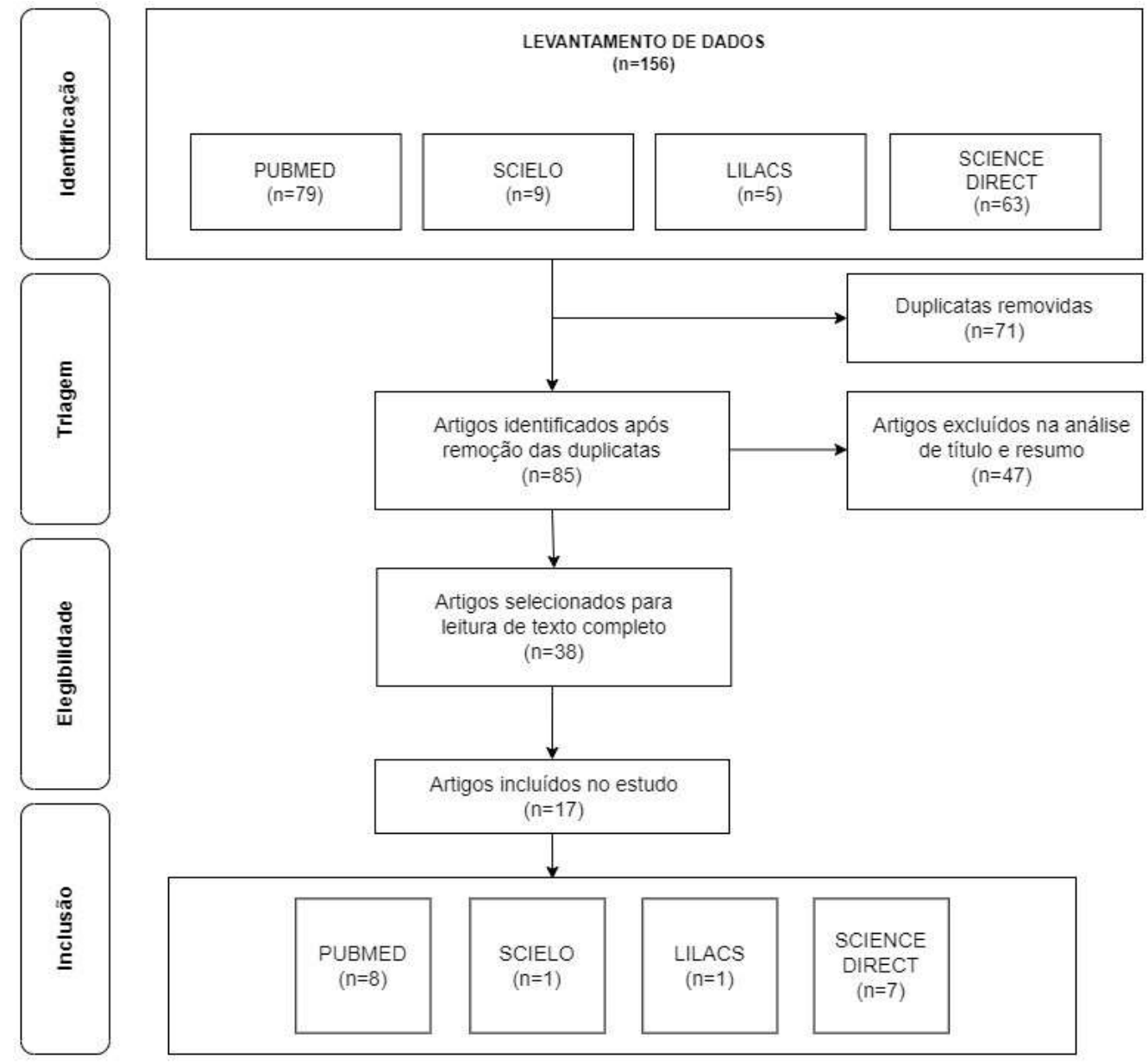

Fonte: Autores.

\section{Resultados e Discussão}

Estudos apontaram uma relação direta entre miRNAs e os estágios de desenvolvimento do câncer cervical (Rao et al., 2012; Sharma el al., 2014). Alguns miRNAs têm localização em região cromossômica frágil, na qual ocorre a integração do DNA viral, dessa forma, proteínas codificadas pelo HPV, como E6 e E7, são capazes de modular a expressão de miRNAs (Chaiwongkot et al., 2013).

Deftereos et al. (2011) relataram que os níveis de miR-21 aumentaram progressivamente de acordo com o grau de lesões intraepiteliais e estavam ainda mais elevados em células de câncer cervical. Além disso, Shishodia et al. (2015) observaram que, no colo do útero, níveis alterados de miR-21 estavam associados a expressão e ativação de STAT3, regulador de genes antiapoptóticos e de controle do ciclo celular. 
A superexpressão de miR-135a em células de câncer cervical se destaca quando comparada a sua expressão em células com características pré-malignas. Os efeitos observados se devem a ação inibitória do miR-135a em seu gene-alvo E3 ubiquitina-proteína ligase, regulando a via de sinalização Wnt/ $\beta$-catenina, favorecendo a transformação, além da migração, invasão e proliferação celular (Leung et al., 2014).

Foi demonstrado que a superexpressão de miR-34a-5p inibiu significativamente a viabilidade, migração e invasão de células de câncer cervical (HeLa), além de promover a apoptose celular. Já a supressão de miR-34a-5p mostrou efeitos contrários, os níveis de expressão de mRNA e proteína de Bcl-2 em células HeLa foram aumentados, gerando um aumento do potencial de viabilidade, migração e invasão dessas células (Wang et al, 2018).

Determinados miRNAs têm regulação epigenética negativa por hipermetilação do DNA, como os genes codificadores de miR-432, miR-1286, miR-641, miR-1290, miR-1287 e miR-95, nos quais se observou a hipermetilação em células infectadas por HPV no colo do útero (Yao et al., 2013). A hipermetilação anormal de miRNAs pode ser analisada por PCR específico de metilação e utilizada para diagnóstico em pacientes com suspeita de câncer cervical (Banno et al., 2014).

Em tumores cervicais e linhas de células de câncer cervical, SiHa e HeLa, a regulação positiva de miR-138 foi capaz de inibir a proliferação e invasão do câncer in vitro e o crescimento do xenotransplante in vivo. Assim, o miR-138 pode ser um supressor de tumor e potencial biomarcador de prognóstico no câncer cervical (Li et al., 2017).

Os miRNAs secretados no soro podem ser usados no diagnóstico e prognóstico de câncer, incluindo alterações em seus níveis séricos em metástases precoces de câncer cervical (Wang \& Chen, 2019). Diversos miRNAs, como miRNA-20a, miR-1246, miR-2392, miR-3147, miR-3162-5p e miR-4484, foram identificados como promissores biomarcadores para detecção de metástase de linfonodo em pacientes com neoplasia cervical (Chen et al., 2013; Zhao et al., 2013). Além desses, também foi descrito o aumento do nível sérico de miR-21 associado as mesmas características metastáticas (Zhang et al., 2016).

Recentemente, Barquet-Muñoz et al. (2022) buscando traçar um perfil de miRNAs associados à metástase linfonodal em pacientes com câncer cervical no estágio inicial, através da avaliação por microarray, identificou-se a superexpressão dos seguintes miRNAs: miR-92b-5p, miR-483-5p, miR-4534 e miR-548ac, que se destacam por seu papel prognóstico.

Mou et al. (2016) determinaram a expressão do miR-148b em células epiteliais cervicais imortalizadas HPV-16 CRL2614 e em células HeLa, e observaram que o miR-148b foi capaz de reduzir a proliferação e invasão celular, além de induzir a apoptose. Também foi observado que esse atua como um supressor de tumor, induzindo a parada do ciclo das células de câncer cervical em G1/S, surgindo como nova abordagem terapêutica.

A variação nos níveis de expressão de miRNAs pode interferir na sensibilidade à quimioterapia. Foi descoberto que o miR-155 suprime a transição epitelial-mesenquimal, que promove a progressão tumoral do câncer cervical, e aumenta a quimiossensibilidade à cisplatina, agente antineoplásico (Leiet al., 2012). Outro miRNA que desempenha um papel importante na resposta quimioterápica é o miR-214, que demonstrou aumentar a sensibilidade à cisplatina através da inibição direta da expressão de Bcl212 em células HeLa e C-33A de câncer cervical (Wang et al., 2013).

Liu et al. (2016) demonstraram que sete miRNAs, miR-642a, miR-142, miR-101-1, miR-3607, miR-502, miR-378c e miR-150, tiveram efeito protetor na sobrevivência de pacientes com câncer cervical. Esses miRNAs podem interferir no prognóstico por meio das vias de sinalização MAPK, P53 e VEGF, o que sugere a aplicação de terapias direcionadas para as três vias, a única via alvo de terapias utilizada, atualmente, é a via de sinalização do VEGF.

Além disso, com o intuito de analisar se os miRNAs presentes na urina poderiam ser usados como biomarcadores confiáveis para diagnóstico e prognóstico do câncer cervical, Aftab et al. (2021) concluíram que a combinação entre miR-1455p, miR-218-5p e miR-34a-5p presentes na urina apresentou 100\% de sensibilidade e 92,8\% de especificidade na distinção entre pacientes controle e pacientes com câncer. 


\section{Conclusão}

As aplicações dos miRNAs são relativamente novas, mas suas possíveis utilidades clínicas estão sendo cada vez mais estimadas. Os padrões nos níveis de expressão de miRNAs em pacientes com câncer cervical e indivíduos saudáveis podem ser utilizados como biomarcadores no diagnóstico do câncer em estágios iniciais, assim como seus usos para avaliação prognóstica e previsão de resultados específicos. Outra potencial aplicação é o tratamento personalizado específico a partir do perfil dos miRNAs.

Dessa forma, a análise das assinaturas de miRNAs em pacientes como câncer cervical surge como promissora ferramenta para o seu diagnóstico, prognóstico e tratamento, sendo necessária a associação desses aos métodos usuais para um melhor manejo clínico.

É de fundamental importância o desenvolvimento de estudos mais aprofundados acerca dessas moléculas, para uma maior elucidação e compreensão dos mecanismos gênicos e de sua aplicabilidade.

\section{Referências}

Aftab, M., Poojary, S. S., Seshan, V., Kumar, S., Agarwal, P., Tandon, S., \& Das, B. C. (2021). Urine miRNA signature as a potential non-invasive diagnostic and prognostic biomarker in cervical cancer. Scientific Reports, 11(1), 1-13.

Banno, K., Iida, M., Yanokura, M., Kisu, I., Iwata, T., Tominaga, E., \& Aoki, D. (2014). MicroRNA in cervical cancer: OncomiRs and tumor suppressor miRs in diagnosis and treatment. The Scientific World Journal, 2014.

Barquet-Muñoz, S. A., Pedroza-Torres, A., Perez-Plasencia, C., Montaño, S., Gallardo-Alvarado, L., Pérez-Montiel, D., \& Cantú-de León, D. (2022). microRNA Profile Associated with Positive Lymph Node Metastasis in Early-Stage Cervical Cancer. Current Oncology, 29(1), $243-254$.

Chaiwongkot, A., Vinokurova, S., Pientong, C., Ekalaksananan, T., Kongyingyoes, B., Kleebkaow, P., \& von Knebel Doeberitz, M. (2013). Differential methylation of E2 binding sites in episomal and integrated HPV 16 genomes in preinvasive and invasive cervical lesions. International journal of cancer, 132(9), 2087-2094.

Chen, J., Yao, D., Li, Y., Chen, H., He, C., Ding, N., \& Long, F. (2013). Serum microRNA expression levels can predict lymph node metastasis in patients with early-stage cervical squamous cell carcinoma. International journal of molecular medicine, 32(3), 557-567.

Deftereos, G., Corrie, S. R., Feng, Q., Morihara, J., Stern, J., Hawes, S. E., \& Kiviat, N. B. (2011). Expression of mir-21 and mir-143 in cervical specimens ranging from histologically normal through to invasive cervical cancer. PloS one, 6(12), e28423.

Hasanzadeh, M., Movahedi, M., Rejali, M., Maleki, F., Moetamani-Ahmadi, M., Seifi, S., \& Avan, A. (2019). The potential prognostic and therapeutic application of tissue and circulating microRNAs in cervical cancer. Journal of cellular physiology, 234(2), 1289-1294.

Hull, R., Mbele, M., Makhafola, T., Hicks, C., Wang, S. M., Reis, R. M., \& Dlamini, Z. (2020). Cervical cancer in low and middle-income countries. Oncology Letters, 20(3), 2058-2074.

Iorio, M. V., \& Croce, C. M. (2012). MicroRNA dysregulation in cancer: diagnostics, monitoring and therapeutics. A comprehensive review. EMBO molecular medicine, 4(3), 143-159.

Lei, C., Wang, Y., Huang, Y., Yu, H., Huang, Y., Wu, L., \& Huang, L. (2012). Up-regulated miR155 reverses the epithelial-mesenchymal transition induced by EGF and increases chemo-sensitivity to cisplatin in human Caski cervical cancer cells. PLoS One, 7(12), e52310.

Leung, C. O., Deng, W., Ye, T. M., Ngan, H. Y., Tsao, S. W., Cheung, A. N., \& Yeung, W. S. (2014). miR-135a leads to cervical cancer cell transformation through regulation of $\beta$-catenin via a SIAH1-dependent ubiquitin proteosomal pathway. Carcinogenesis, 35(9), 1931-1940.

Li, H., Sheng, Y., Zhang, Y., Gao, N., Deng, X., \& Sheng, X. (2017). MicroRNA-138 is a potential biomarker and tumor suppressor in human cervical carcinoma by reversely correlated with TCF3 gene. Gynecologic oncology, 145(3), 569-576.

Liu, B., Ding, J. F., Luo, J., Lu, L., Yang, F., \& Tan, X. D. (2016). Seven protective miRNA signatures for prognosis of cervical cancer. Oncotarget, 7(35), 56690 .

Marth, C., Landoni, F., Mahner, S., McCormack, M., Gonzalez-Martin, A., \& Colombo, N. (2017). Cervical cancer: ESMO Clinical Practice Guidelines for diagnosis, treatment and follow-up. Annals of Oncology, 28, iv72-iv83.

Mou, Z., Xu, X., Dong, M., \& Xu, J. (2016). MicroRNA-148b acts as a tumor suppressor in cervical cancer by inducing G1/S-phase cell cycle arrest and apoptosis in a caspase-3-dependent manner. Medical science monitor: international medical journal of experimental and clinical research, $22,2809$.

Pardini, B., De Maria, D., Francavilla, A., Di Gaetano, C., Ronco, G., \& Naccarati, A. (2018). MicroRNAs as markers of progression in cervical cancer: a systematic review. BMC cancer, 18(1), 1-17.

Qiu, H., Liang, D., Liu, L., Xiang, Q., Yi, Z., \& Ji, Y. (2020). A novel circulating miRNA-based signature for the diagnosis and prognosis prediction of earlystage cervical cancer. Technology in Cancer Research \& Treatment, 19, 1533033820970667. 
Research, Society and Development, v. 11, n. 2, e25711225776, 2022

(CC BY 4.0) | ISSN 2525-3409 | DOI: http://dx.doi.org/10.33448/rsd-v11i2.25776

Rao, Q., Zhou, H., Peng, Y., Li, J., \& Lin, Z. (2012). Aberrant microRNA expression in human cervical carcinomas. Medical oncology, 29(2), $1242-1248$.

Sharma, G., Dua, P., \& Mohan Agarwal, S. (2014). A comprehensive review of dysregulated miRNAs involved in cervical cancer. Current genomics, 15(4), 310-323.

Shishodia, G., Shukla, S., Srivastava, Y., Masaldan, S., Mehta, S., Bhambhani, S., \& Bharti, A. C. (2015). Alterations in microRNAs miR-21 and let-7a correlate with aberrant STAT3 signaling and downstream effects during cervical carcinogenesis. Molecular cancer, 14(1), 1-13.

Silva, A. B. L. P., Feitosa, A. A., Júnior, A. A. S. G., da Cruz Diniz, C. B., de Brito, C. A., Gonçalves, I. C., \& de Sousa Fideles, L. Perfil epidemiológico do câncer do colo do útero no estado do Piauí. Perfil epidemiológico do câncer do colo do útero no estado do Piauí, 1-388.

Souza, M. T. D., Silva, M. D. D., \& Carvalho, R. D. (2010). Revisão integrativa: o que é e como fazer. Einstein, 8, 102-106.

Vaz, G. P., Bitencourt, E. L., Martins, G. S., de Carvalho, A. A. B., da Costa, S. B., \& Júnior, P. M. R. (2020). Perfil epidemiológico do câncer de colo de útero no estado do Tocantis no período de 2013 A 2019. Revista de Patologia do Tocantins, 7(2), 114-117.

Wang, F., Liu, M., Li, X., \& Tang, H. (2013). MiR-214 reduces cell survival and enhances cisplatin-induced cytotoxicity via down-regulation of Bcl212 in cervical cancer cells. FEBS letters, 587(5), 488-495.

Wang, J. Y., \& Chen, L. J. (2019). The role of miRNAs in the invasion and metastasis of cervical cancer. Bioscience reports, 39 (3), BSR20181377.

Wang, X., Xie, Y., \& Wang, J. (2018). Overexpression of microRNA-34a-5p inhibits proliferation and promotes apoptosis of human cervical cancer cells by downregulation of Bcl-2. Oncology research, 26(6), 977.

Yao, T., Rao, Q., Liu, L., Zheng, C., Xie, Q., Liang, J., \& Lin, Z. (2013). Exploration of tumor-suppressive microRNAs silenced by DNA hypermethylation in cervical cancer. Virology journal, 10(1), 1-7.

Zhang, L., Zhan, X., Yan, D., \& Wang, Z. (2016). Circulating microRNA-21 is involved in lymph node metastasis in cervical cancer by targeting RASA1. International Journal of Gynecologic Cancer, 26(5).

Zhao, S., Yao, D., Chen, J., \& Ding, N. (2013). Circulating miRNA-20a and miRNA-203 for screening lymph node metastasis in early stage cervical cancer. Genetic testing and molecular biomarkers, 17(8), 631-636. 